\title{
Erratum to: Proposal for correction of the SCR calculation bias in Solvency II
}

\author{
Martin Hampel • Dietmar Pfeifer
}

Published online: 1 May 2014

(C) Springer-Verlag Berlin Heidelberg 2014

Erratum to: ZVersWiss (2011) 100:733-743

DOI 10.1007/s12297-011-0174-y

Page 738, Sect. 3 Add the general assumption $u \in(1 / 2,1)$ for the remainder of the article (Sect. 3 and the appendix).

Page 739, Line 5: The condition $\tilde{s}^{2} \in \mathbb{R}$ is not correct: Define $x_{+,-}:=q_{u} \pm$ $\sqrt{-2 \ln \left(F_{m, s^{2}}^{-1}(u)\right)+q_{u}^{2}}$ and replace $\tilde{s}^{2} \in \mathbb{R}$ by the condition $x_{+} \in \mathbb{R}$ in case of $\tilde{s}^{2}=$ $\exp \left(x_{+}^{2}\right)-1$ and $x_{-} \in \mathbb{R}_{0}^{+}$in case of $\tilde{s}^{2}=\exp \left(x_{-}^{2}\right)-1$, respectively. Note that $x_{+}$is always non-negative if it is real (since $q_{u}>0$ holds for $u \in(1 / 2,1)$ ). In the further analysis we focused on the existence of at least one solution. Therefore replace $\tilde{s}^{2} \in \mathbb{R}$ or any variant there by $x_{+} \in \mathbb{R}$ if the term $\tilde{s}^{2} \in \mathbb{R}$ or its variant (implicitly) stands for the replaced condition of Theorem 2. In particular, this impacts the appendix. Nevertheless, the proving argumentation in the appendix still works.

Page 739, Line 13 and Line 15: Take into account that $x$ is a non-negative real number.

Page 740, Line 4 and Lemma 2: Add "if $u$ is 0.995" at the end of Line 4. Also, add $u=0.995$ as formal assumption in the assertion of Lemma 2.

Page 742, Line 7: Replace "u-quantile" by "quantile".

The online version of the original article can be found under doi:10.1007/s12297-011-0174-y

Dipl.-Math. M. Hampel ( $\varangle)$ · Prof. Dr. D. Pfeifer Department of Mathematics, Carl von Ossietzky University Oldenburg, Carl von Ossietzky Straße 9-11, 26111 Oldenburg, Germany e-mail: Martin.Hampel@uni-oldenburg.de 


\section{Reference}

Hampel, M., Pfeifer, D.: Proposal for correction of the SCR calculation bias in Solvency II. ZVersWiss. 100, 733-743 (2011) 